\title{
Antihypertensive Treatment and Kidney Function in Routine Practice in Patients with Type 2 Diabetes Mellitus: The Results of the Prospective "The Scythian" Trial in Ukraine
}

\author{
Dmitri D. Ivanov ${ }^{*}, 1$ and Boris N. Mankovsky² \\ ${ }^{I}$ Dept. of Nephrology and Renal Replacement Therapy, ${ }^{2}$ Dept. of Diabetology, National Medical Academy of \\ Postgraduate Education named by P.L. Schupik, Kiev, Ukraine
}

\begin{abstract}
Background: Hypertension, albuminuria and decreased GFR are regarded as independent risk factors that reflect progression of the diabetic kidney disease (DKD). We carried out second phase of prospective study which aim was to learn the possibility of implementation of intensive antihypertensive therapy into the routine clinical practice and assessment of such treatment influence on kidney function in patients with type 2 diabetes mellitus (T2DM).

Methods: In the study 637 patients were included with DKD and arterial hypertension with BP level more than 130/80 $\mathrm{mmHg}$ and less than $180 / 100 \mathrm{mmHg}$, having chronic kidneys disease (CKD) 1-4. As a basis for intensive antihypertensive treatment fixed combination of perindopril $5 \mathrm{mg}$ and indapamide $1.25 \mathrm{mg}$ were prescribed. If needed, after 4 weeks the dosage was increased up to perindopril $10 \mathrm{mg}$ / indapamide $2.5 \mathrm{mg}$ to achieve targeted BP less that $130 / 80 \mathrm{mmHg}$. If targeted BP had not been achieved other antihypertensive preparations were added.

Results: After 4 weeks of treatment average levels of systolic and diastolic BP were $141.3 \pm 0.5$ and $86.3 \pm 0.3$, after 8 weeks of therapy - 131.6 \pm 0.4 and $81.3 \pm 0.3 \mathrm{mmHg}$, and after 12 weeks of treatment $-127.2 \pm 0.3$ and $78.7 \pm 0.2 \mathrm{mmHg}$ respectively. Target BP was achieved in 489 patients (73.3\%) during 12 weeks of treatment. Statistically significant increase of GFR as a result of 12 weeks of antihypertensive therapy with fixed perindopril/indapamide combination was found to be between $84.3 \pm 1.1$ and $94.7 \pm 1.1 \mathrm{ml} / \mathrm{min} . / 1,73 \mathrm{~m}^{2}, \mathrm{p}<0.01$.

Conclusion: Antihypertensive therapy with fixed combination of perindopril 5-10 mg/indapamide 1.25-2.5 mg introduced into routine clinical practice of T2DM patients' treatment, is effective for BP reduction, achievement of its targeted values and resulted in statistically significant trend to kidney function improvement.
\end{abstract}

Keywords: Combined antihypertensive therapy, Diabetic kidney disease (DKD), glomerular filtration rate (GFR), hypertension, microalbuminuria.

\section{INTRODUCTION}

Kidneys impairment is one of the most serious manifestations of diabetic microangiopathies [1]. Hypertension, albuminuria and decreased GFR are regarded as independent risk factors that reflect progression of the diabetic kidney disease (DKD) [2]. According to KDOQI Clinical Practice Guidelines and Clinical Practice Recommendations for Diabetes and Chronic Kidney Disease, most people with diabetes and CKD have hypertension and treatment of hypertension slows the progression of CKD [3]. According to 2012 ADA recommendations [4], to reduce the risk or slow the progression of nephropathy, patients need to optimize their glucosecontrol (A) and BP control (A) with an ACEinhibitors (ACEI) or angiotensin II receptor blockers (ARB), usually in combination with a diuretics.

*Address correspondence to this author at the Dept. of Nephrology and Renal Replacement Therapy, National Medical Academy of Postgraduate Education named by P.L. Schupik, Kiev, Ukraine; Tel: +380504448788 ; Fax: +380442846897; E-mail: ivanovdd@i.kiev.ua
To determine epidemiologic prevalence of clinicallaboratory manifestations of DKD, diagnosed by KDOQI criteria [3], in patients with T2DM and to assess antihypertensive treatment efficacy in Ukraine with national population above 46 million, open label, prospective randomize cross-sectional trial "The Scythian" was carried out. The study design had 2 stages. The first one (20082009) investigated epidemiologic prevalence and severity of renal damage in T2DM patients (Fig. 1). The second stage (2010-2011) studied the efficacy of ACEI+ potential for treatment of diabetic nephropathy.

According to stage 1 results of "The Scythian" trial changes in GFR as hypo- or hyperfiltration was shown in 8 out of 10 patients with T2DM [5]. Elevation of BP above $130 / 80$ and particularly above $140 / 90 \mathrm{mmHg}$ precedes increase/reduction in FGR. Micro albuminuria takes third place by time of its appearance among informative diabetic kidney disease signs [5].

The present work presents the results of $2^{\text {nd }}$ stage of the trial "The Scythian", which aimed to study the possibility of intensive antihypertensive treatment to be introduced into the routine clinical practice and assessment of such therapy 
effect on kidney function in patients with T2DM. The primary endpoint was to achieve target blood pressure (BP) less than $130 / 80 \mathrm{mmHg}$ with fixed combination of perindopril/indapamide. Secondary endpoint was to ascertain renal function trend by estimating GFR.

\section{DKD In Patients With Diabetes Type 2 In Ukraine: "The Scythian" trial}

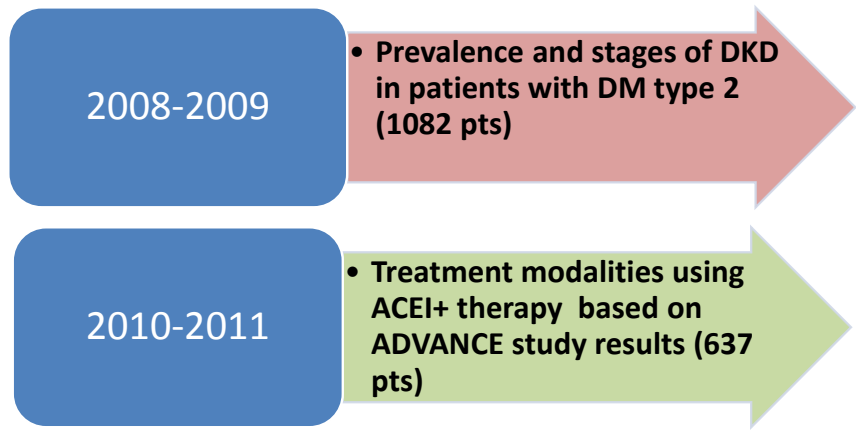

Fig. (1). Stages of "The Scythian" trial.

\section{SUBJECTS AND METHODS}

637 patients with T2DM were included in prospective study. Inclusion criteria were as follows: established diagnosis of T2DM, age of more than 40 years and diagnosed arterial hypertension of $1-2^{\text {nd }}$ grades with BP level more than 130/80 $\mathrm{mmHg}$ and less than $180 / 100 \mathrm{mmHg}$. Exclusion criteria were: arterial hypertension of $3^{\text {rd }}$ stage, BP level of 180/110 $\mathrm{mmHg}$ and more, diagnosed type 1diabetes mellitus, age less than 40 years, history of myocardial infarction, cerebrovascular event in previous 6 months before examination, polycystic kidney disease presence, oncological diseases, pregnancy or breastfeeding, anemia at hemoglobin level less than $90 \mathrm{~g} / \mathrm{L}$.

As a basis for intensive antihypertensive treatment fixed combination of perindopril $5 \mathrm{mg}+$ indapamide $1.25 \mathrm{mg}$ was selected. The reason for selection of this combination were the results of the ADVANCE, where in large population of patients (more than 11 thousand patients with T2DM) it was demonstrated that this prescription at the background of standard antihypertensive therapy allowed to reduce allcause mortality by $14 \%$, cardiovascular mortality by $18 \%$, and renal diabetes complications development by $21 \%$ [6].

The study was carried out according to the algorithm shown at Fig. (2) with providing patient's signed informed consensus. In a case of BP level more than $130 / 80 \mathrm{mmHg}$ the patients were prescribed fixed combination of perindopril $5 \mathrm{mg}+$ indapamide $1.25 \mathrm{mg}$ once a day in the morning. In case of absence of initial antihypertensive therapy the fixed combination was prescribed in monotherapy regimen. If the patients were receiving calcium channel blockers $(\mathrm{CCB})$ or $\beta$-blockers $(\beta \mathrm{Bs})$, fixed combination was prescribed additionally. If patients were initially receiving ACEI, ARB, or combination which include ACEI or ARB this treatment was discontinued and fixed combination of perindopril $5 \mathrm{mg}$ + indapamide $1.25 \mathrm{mg}$ was prescribed. BP measurement was performed in 4 weeks after commencement of perindopril/ indapamide combination treatment. In case BP level achieved targeted level less than $130 / 80 \mathrm{mmHg}$ the patient was recommended to continue treatment in the same dose. IfBP levels were raised, the dosages were increased twofold up to $10 \mathrm{mg}$ of perindopril and $2.5 \mathrm{mg}$ of indapamide. In 8 weeks BP measurements were repeated and when it was

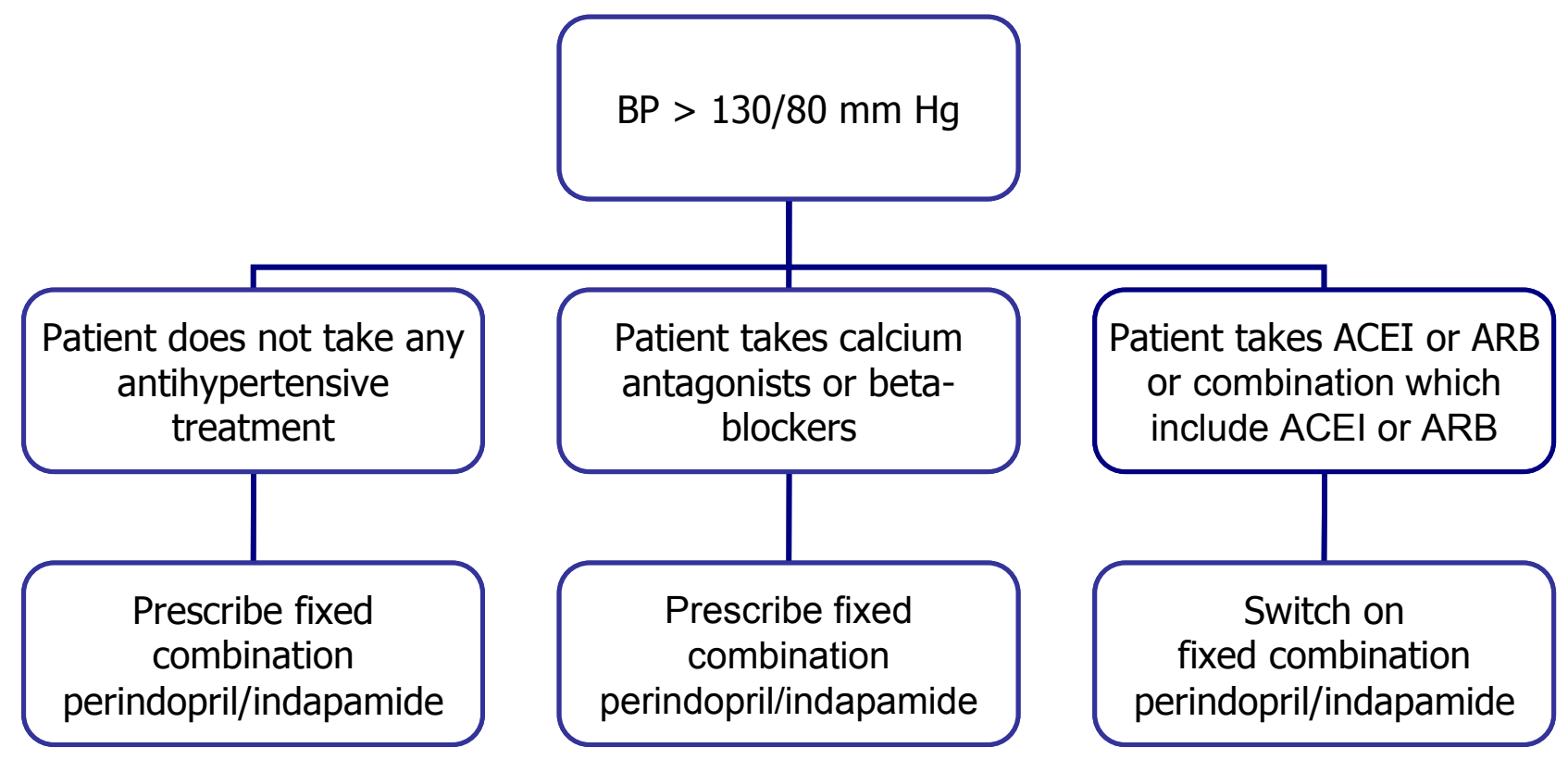

\section{Perindopril/indapamide doses titration every 4 weeks till targeted BP $<130 / 809 \mathrm{~mm} \mathrm{Hg}$.}

Fig. (2). Algorithm of antihypertensive treatment during $2^{\text {nd }}$ stage of "The Scythian" trial. 
necessary other antihypertensive preparations were additionally prescribed to achieve targeted BP below 130/80 $\mathrm{mmHg}$. BP levels were evaluated by office measurements in three consecutive days.

In 12 weeks after trial initiation measurements of BP level were provided to the patients and GFR was calculated. GFR index was calculated on the basis of serum creatinine levels by the Cockcroft \& Gault formula [7]. The serum creatinine was measured using the modified Jaffe method in local certificated labs.

There were $289(45.4 \%)$ male and 348 (54.6\%) female patients includedin this trial. The mean age of the patients was $58 \pm 0.6$ years $(\mathrm{M} \pm \mathrm{SD})$, average diabetes duration $7 \pm 0.3$ years. Baseline creatinine level was $101 \pm 1.2 \mu \mathrm{mol} / \mathrm{L}$, GFR - $84 \pm 1.1 \mathrm{~mL} / \mathrm{min} . / 1.73 \mathrm{~m}^{2}$. Mean baseline systolic BP was $162 \pm 0.5 \mathrm{mmHg}$, mean baseline diastolic BP - 95 \pm 0.3 mmHg. $48 \%$ of patients has macroalbuminuria, $52 \%$ microalbuminuria detected by dipstick tests.

The majority of patients received antihypertensive therapy at the baseline: 452 patients $(67.8 \%)$ were treated with ACEI, 58 patients $(8,7 \%)$ were taking ARB, 171 $(25,6 \%)$ patients- $\mathrm{CCB}, 218(32,7 \%)$ patients - $\beta \mathrm{B}, 235$ $(35,2 \%)$ patients - diuretics, 26 patients $(3,9 \%)-\alpha$-blockers. However, only 340 patients $(51 \%)$ received antihypertensive preparations regularly. The majority of patients $(61.6 \%)$ received combined antihypertensive therapy. Low sodium diet was recommended to everybody.

Obesity, defined as body mass index more than $30 \mathrm{~kg} / \mathrm{m}^{2}$ in both genders, was reported in $386(53 \%)$ of patients, 289 (43.5\%) patients had excess body mass with body mass index more than $25 \mathrm{~kg} / \mathrm{m}^{2}$.

Absolute majority of patients (563 patients - 86.9\%) received glucose lowering drugsin tablets for hyperglycemia correction, while insulin therapy was prescribed for 60 patients $(9.2 \%)$ and 25 persons $(3.9 \%)$ received combined treatment with glucose lowering tableted drugs and insulin.

Statistical analysis of data received was carried out using online Creative Research Systems calculator of statistical significance [8]. The Microsoft PowerPoint 2010 and Microsoft Excel were used to tabulate and process the data.

\section{RESULTS}

As result of the treatment provided significant reduction of systolic and diastolic BP was achieved (Figs. 3, 4). In 4 weeks of treatment average systolic and diastolic blood pressure were $141,3 \pm 0.5$ and $86.3 \pm 0.3 \mathrm{mmHg}$, in 8 weeks of therapy $-131.6 \pm 0,4$ and $81.3 \pm 0.3 \mathrm{mmHg}$, and in 12 weeks of treatment $-127.2 \pm 0.3$ and $78.7 \pm 0.2 \mathrm{mmHg}$, respectively. In absolute numbers systolic BP reduction for the period of treatment was $34.7 \mathrm{mmHg}$, diastolic $\mathrm{BP}$ reduction -16.5 $\mathrm{mmHg}, \mathrm{p}<0.001$, compared to the baseline level before start of the therapy with fixed combination of perindopril/indapamide. It is important to emphasize that at the end of follow-up period among patients $(n=353)$, receiving gperindopril $5 \mathrm{mg}$ /indapamide $1.25 \mathrm{mg}$ as monotherapy in a dose of 1 tablet a day average arterial pressure values were $128.5 \pm 0.6$ and $79.9 \pm 0.5 \mathrm{mmHg}$, while in those who received perindopril $10 \mathrm{mg} /$ indapamide $2.5 \mathrm{a}$ day $(n=284)$ the values of systolic and diastolic BP were $123.4 \pm 0.5$ and $76.7 \pm 0.4 \mathrm{mmHg}$, respectively.

In absolute numbers in the group of patients, receiving daily perindopril $5 \mathrm{mg}$ /indapamide $1.25 \mathrm{mg}(\mathrm{n}=353)$, reductions of systolic and diastolic BP for the time of treatment were $33.7 \mathrm{mmHg}$ and $17 \mathrm{mmHg}$, respectively, $\mathrm{p}<0,001$, compared to the baseline. In the group of patients who took daily perindopril $10 \mathrm{mg}$ /indapamide $2.5 \mathrm{mg}(\mathrm{n}=284)$, reductions of systolic and diastolic BP for the time of treatment were 38.4 $\mathrm{mmHg}$ and $15.8 \mathrm{mmHg}$, respectively, $\mathrm{p}<0,001$, compared to the baseline.

Target BP below 130/80 mmHg (according to guidelines $[4,9])$ was achieved in 171 patients $(25.6 \%)$ in 4 weeks; in 373 patients $(55.9 \%)$ in 8 weeks after treatment initiation, and in $489(73.3 \%)$ patients in 12 weeks of treatment arterial BP sustained in targeted ranges. So, 148 patients $(26,7 \%)$ needed to add/increase $\mathrm{CCB}$ or $\beta \mathrm{B}$ to reach targeted $\mathrm{BP}$ after 12 weeks of perindopril/indapamide. Among the patients with baseline BP level below $160 / 100 \mathrm{mmHg}$, at the end of 12-week follow-up period targeted BP were reported in $87.5 \%$ of patients $(n=557)$, and among persons, with baseline BP level above 160/100 $\mathrm{mmHg}$ targeted BP levels were achieved in $72.5 \%(\mathrm{n}=462)$.

Targeted BP at the end of 12-week observation period was achieved in $78.1 \%$ of patients $(n=497)$ that initially received ACE inhibitors, in $81.8 \%$ of patients $(n=521)$ that previously received angiotensin II receptor blockers, in $74.1 \%$ of patients $(n=472)$ previously treated with $C C B$ and in $95.7 \%$ of persons $(n=610)$ that received $\beta B$.

At the beginning of the study $57,3 \%$ of patients (380) used perindopril/indapamide in combination with other antihypertensive drugs while at the end of trial $-47,5 \%$ (313).

Adequate antihypertensive therapy led to positive GRF and albuminuria modifications. Among micro albuminuria patients $21 \%$ were transformed in normo albuminuria, while $19 \%$ of that with macro albuminuria changed to micro albuminuria. No data were obtained in absolute numbers. Hence, statistically significant GRF increase under the influence of 12-week antihypertensive therapy with fixed combination of perindopril/indapamide - from $84.3 \pm 1.1$ to $94.7 \pm 1.1 \mathrm{~mL} / \mathrm{min} . / 1,73 \mathrm{~m}^{2}, \mathrm{p}<0.01$ (Fig. 5) was documented. Proportion of patients with GRF more than 30 and less than $60 \mathrm{~mL} / \mathrm{min} . / 1,73 \mathrm{~m}^{2}$ significantly decreased from 17.6 $(n=112)$ to $8.1 \%(n=51)(p<0,001)$. Statistically significant GRF increase under the influence of antihypertensive therapy was seen independently on the initial BP level. However, correlation between BP reduction and initial GRF value was not determined $(\mathrm{r}=0,28)$.

\section{DISCUSSION}

First stage of "The Scythian" trial demonstrated that micro albuminuria is a late sign that follows BP increase and alteration of GFR and characterizes progression of diabetic kidney disease [5]. Urinary microalbumin excretion is only one risk factor for overt macroalbuminuria progression and GFR decline, and other important factors were implicated as important for prevention of ESRD [10]. Achievement of targeted BP values is the most important condition of DKD progression delay $[4,11]$. 


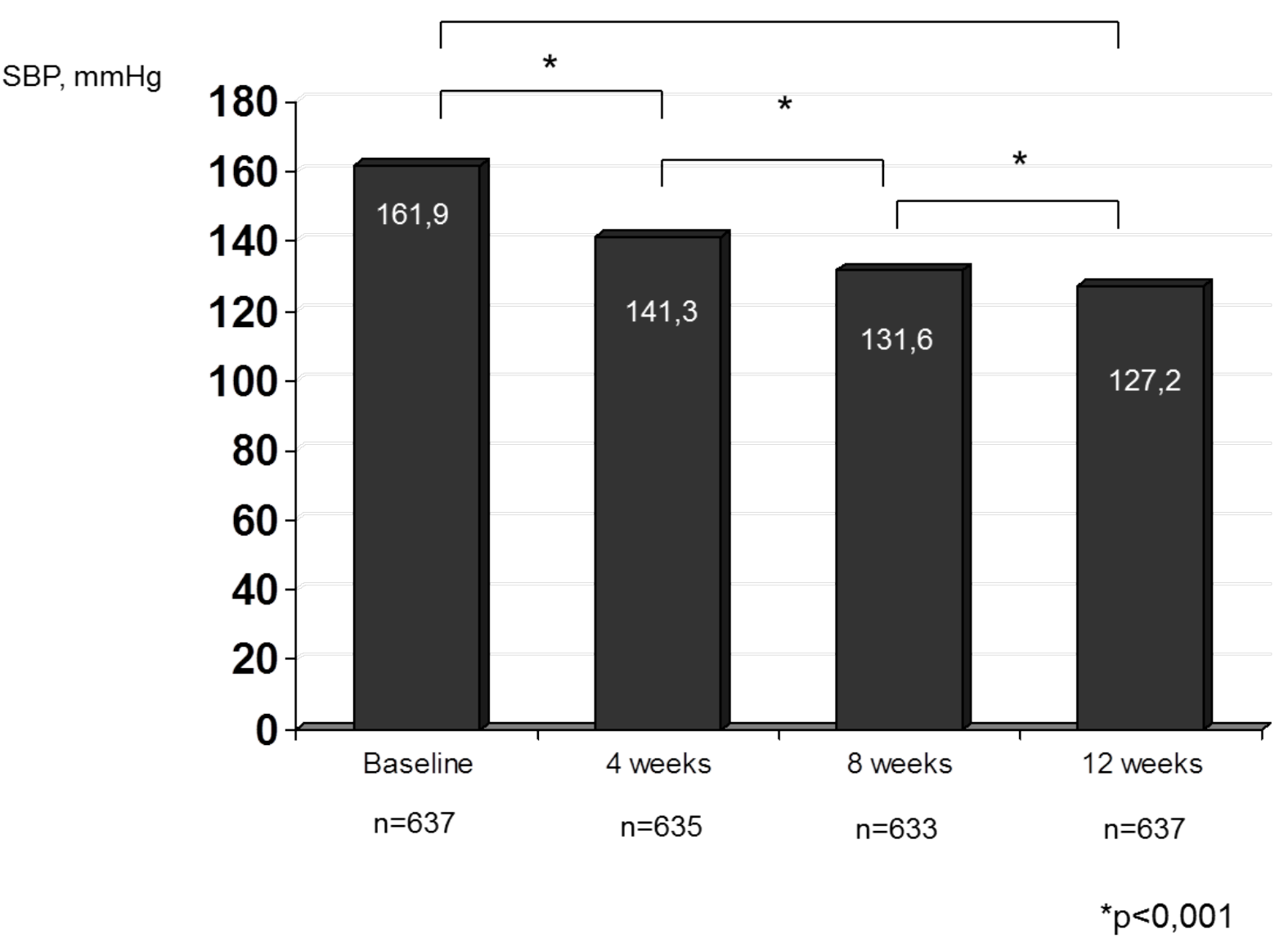

Fig. (3). Dynamics of systolic BP in patients with T2DM treated with fixed combination of perindopril/indapamide .

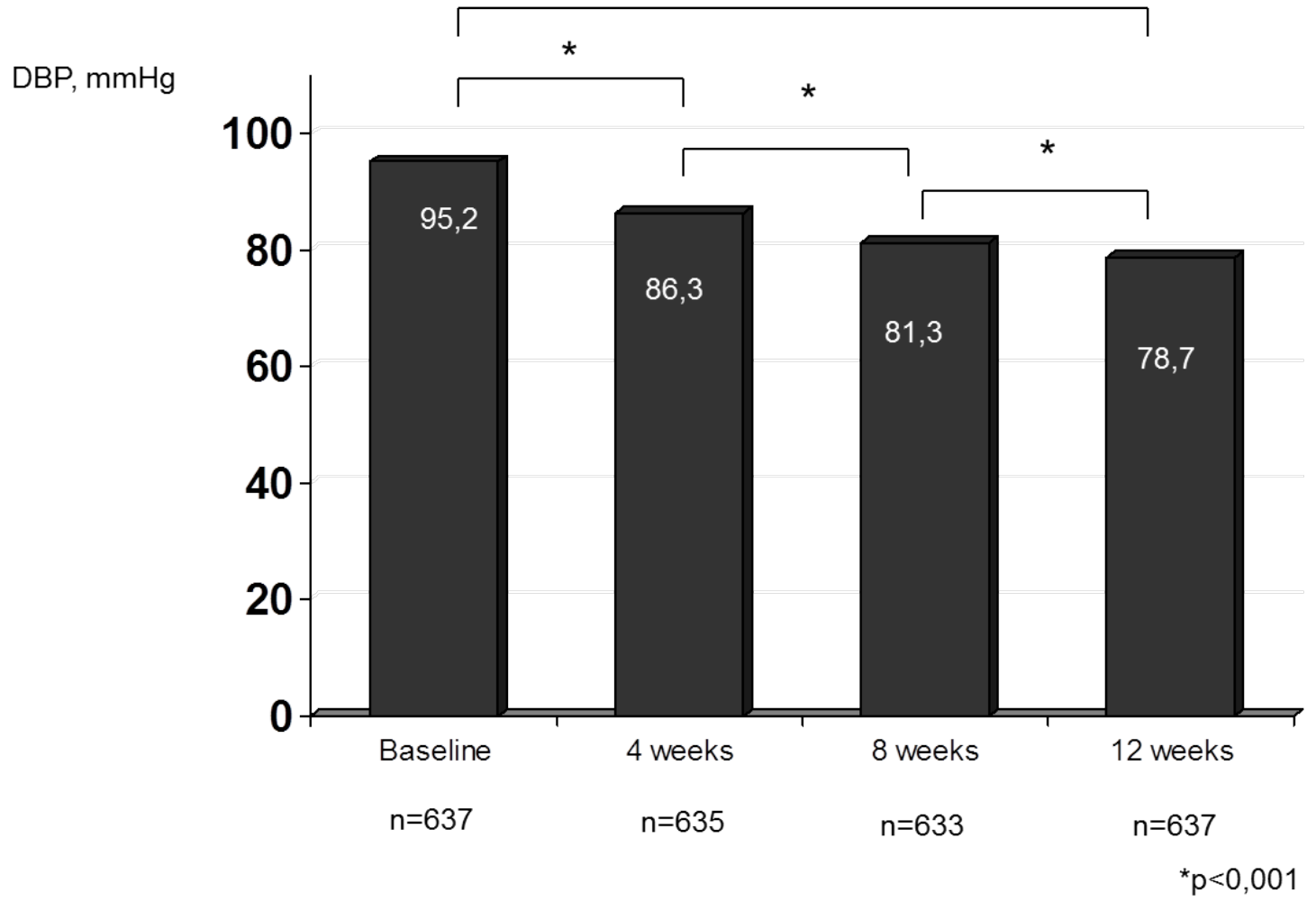

Fig. (4). Dynamics of diastolic BP in patients with T2DM treated with fixed combination of perindopril/indapamide. 


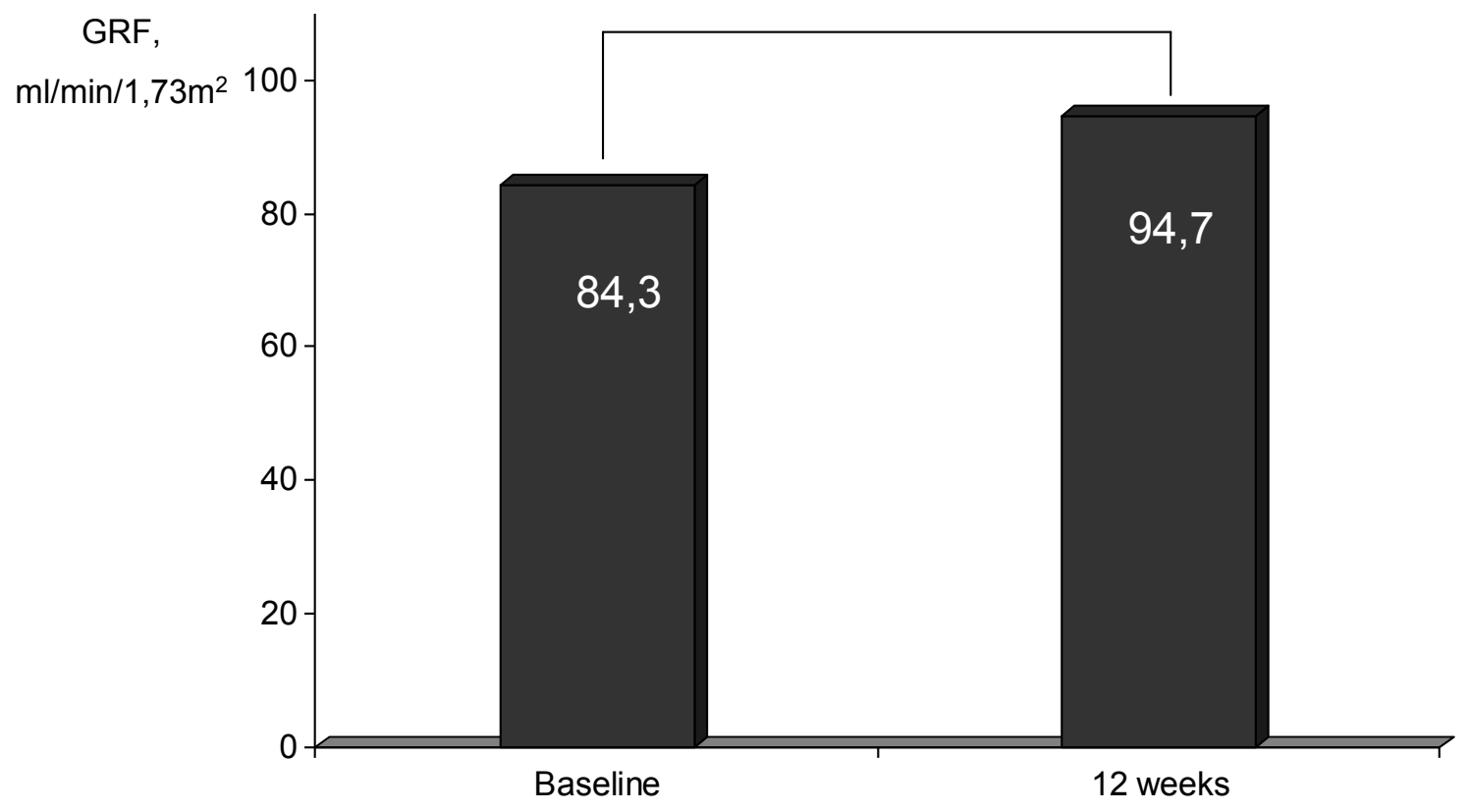

${ }^{*} p<0,001$

Fig. (5). Effect of antihypertensive therapy with fixed combination of perindopril/indapamide on GFR values, $\mathrm{mL} / \mathrm{min} / 1,73 \mathrm{~m}{ }^{2}$.

Based onthe results obtained it maybe possible to state that antihypertensive therapy with fixed combination of perindopril/indapamide led to targeted BP achievement in absolute number of patients with T2DM included in the study. In our opinion, renal damage may have combined influence due to hypertension and diabetic nephropathy. Hence any evidence-based prescription seems to be rational in routine practice demonstrating statistically significant positive trend in GFR with achieving of targeted BP.

In a recent review [12] which included 85 trials and 21708 patients it was shown that development of ESRD and progression of microalbuminuria to macroalbuminuria were reduced significantly with ACEI versus placebo and ARB versus placebo but not with combined therapy with ACEI+ARB versus monotherapy. There was also observed a significant reduction in the risk of nonfatal cardiovascular events with ACEI versus placebo, but not with ARB versus placebo, ACEI versus ARB or with combined therapy with ACEI+ARB versus monotherapy [12]. In connection with results of ADVANCE study [6], and recent review of renal, cardiovascular and mortality endpoints in antihypertensive trials in diabetic patients [13], therapy selection in favor of ACE inhibitor seems to be rational.

It is important to stress that effectiveness of fixed combination of perindopril/indapamide in terms of possibility to achieve the targeted BP values did not depend on previous antihypertensive therapy, being in ranges from $74 \%$ of patients receiving initially CCB to $96 \%$ of patients receiving $\beta B$. At the same time, targeted $\mathrm{BP}$ achievement depended directly on the dose of fixed perindopril/indapamide combination that is known for both ACEI and ARB. In one of the recent meta-analyses of randomized clinical trials, alternate doses of angiotensin receptor blockers or angiotensin converting enzyme inhibitors in patients with T2DM and microalbuminuria were compared. MEDLINE, EMBASE and the Cochrane Register of Controlled Trials were searched from January 2006 to August 2010. The authors support current recommendations to titrate renin-angiotensin inhibitors to maximum dose whilst considering risk of adverse side effects with higher doses [14].

Adequate antihypertensive therapy led to statistically significant GFR increase and reduction of patients with CKD stage 3 under the influence of 12-week antihypertensive therapy with fixed combination of perindopril/indapamide. Positive GFR modifications were accompanied by reduction of relative patient's number that were found to have significant GFR reduction. On the other hand, significant reduction of arterial pressure values was reported independently on the initial GFR value.

The strengths of presented results are the possibility of effective targeting BP in T2DM patients with perindopril/indapamide fixed dose and achieving rapid trend in kidney function renewal. Hence the weakness of the study is the absence of strict control of albuminuria level that does not allow to analyze correlation between microalbuminuria/ creatinine ratio and GFR. With new data obtained it may be reasonable to use GFR-EPI calculator for future trials.

\section{CONCLUSION}

The data obtained in "The Scynthian" trial demonstrate that a reduction to targeted blood pressure in T2DM patients can be achieved in a short period of time based on prescription of fixed perindopril $(5-10 \mathrm{mg})$ /indapamide (1,25-2.5 mg)combination in $73 \%$ of cohort while adding 
$\mathrm{CCB}$ or $\beta \mathrm{B}$ is necessary for the rest one. While targeted $\mathrm{BP}$ grade achieved the GFR is improved globally as well as level of microalbuminuria. In our opinion antihypertensive therapy with fixed perindopril/indapamide combination could be introduced into the routine clinical practice in patients with T2DM and hypertension stage 1 or 2 as effective tool for BP reduction, achievement of BP targeted values and improvement of kidney function.

\section{CONFLICT OF INTEREST}

The clinical trial was conducted with a financial support of Servier company. The authors received lecture fees from Servier.

\section{ACKNOWLEDGEMENTS}

Authors, being project coordinators, express their deep gratitude to the clinicians that participated in the trial.

\section{REFERENCES}

[1] Mogensen CE. Microalbuminuria and hypertension with focus on type 1 and type 2 diabetes. J Int Med 2003; 254: 45-66.

[2] Friedman EA, Friedman AL. Is there really good news about pandemic diabetic nephropathy? Nephrol Dial Transplant 2007; 22: 681-3.

[3] KDOQI clinical practice guidelines and clinical practice recommendations for diabetes and chronic kidney disease. AJKD 2007; 49 (2): S2: 3-154.

[4] Executive summary. standards of medical care in diabetes 2012. Diabetes Care 2012; 35 (Suppl 1): S11-63.
[5] Ivanov D, Mankovsky B. Epidemiology and treatment results of diabetic kidney disease in Ukraine: Clinical Trial "The Scythian", Stage I. BANTAO J 2010; 8 (1): 17-21.

[6] Patel A, MacMahon S, Chalmers J, et al. Effects of a fixed combination of perindopril and indapamide on macrovascular and microvascular outcomes in patients with type 2 diabetes mellitus (the ADVANCE trial): a randomized trial. Lancet 2007; 370: 82940.

[7] Cockcroft DW, Gault MH. Prediction of creatinine clearance from serum creatinine. Nephron 1976; 16: 31-41.

[8] http://www.surveysystem.com/sscalc.htm

[9] Mansia G, De Backer G, Dominiczak A, et al. European Society of Hypertension; European Society of Cardiology. 2007 ESH-ESC Guidelines for the management of arterial hypertension: the task force for the management of arterial hypertension of the European Society of Hypertension (ESH) and of the European Society of Cardiology (ESC). Blood Press 2007; 16(3): 135-232.

[10] Yokoyama H, Kanno S, Takahashi S, et al. Risks for glomerular filtration rate decline in association with progression of albuminuria in type 2 diabetes. Nephrol Dial Transplant 2011; 26: 2924-30.

[11] Plantinga LC, Miller ER III, Stevens LA, et al. Chronic Kidney Disease Surveillance Team for the Centers for Disease Control Prevention. BP control among persons without and with chronic kidney disease. US Trends and Risk Factors 1999-2006. Hypertension 2009. - Published online before print May 26, 2009. doi: 10.1161/HYPERTENSIONAHA.109.129841

[12] Maione A, Navaneethan SD, Graziano G et al. Angiotensinconverting enzyme inhibitors, angiotensin receptor blockers and combined therapy in patients with micro- and macroalbuminuria and cardiovascular risk factors: a systematic review of randomized controlled trials. Nephrol Dial Transplant 2011; 26: 2827-46.

[13] Garcia-Donaire JA, Segura J, Cerezo C, Ruilope LM. A review of renal, cardiovascular and mortality endpoints in antihypertensive trials in diabetic patients. BP 2011;20: 322-34.

[14] Blacklock CL, Hirst JA, Taylor KS, Stevens RJ, Roberts NW, Farmer AJ. Evidence for a dose effect of renin-angiotensin system inhibition on progression of microalbuminuria in Type 2 diabetes: a meta-analysis. Diabet Med 2011; 28: 1182-7. 